\title{
Use of Smartphone Applications in Children with Type 1 Diabetes Mellitus
}

\author{
Rajni Sharma ${ }^{1}$
}

Received: 23 October 2019 / Accepted: 24 October 2019 / Published online: 11 November 2019

(C) Dr. K C Chaudhuri Foundation 2019

Type 1 diabetes mellitus (T1DM) is one of the most common chronic medical disorders in children. The management consists of self-monitoring of blood glucose (BG) levels, lifelong multiple daily injections/pump therapy of insulin, exercise and regulated diet. Good glycemic control (with target HbA1c levels $\leq 7.5$ in children) is essential to prevent long-term macro-vascular complications in the form of retinopathy, neuropathy and nephropathy [1]. However, compliance with this intensive treatment is problematic especially during the transition phase of adolescence and missing insulin doses and BG checks is not uncommon in this age group.

Good adherence with insulin therapy and monitoring of BG is known to be associated with better glycemic control and prevent morbidity, mortality and adverse outcomes [2]. With the increasing number of smartphone users, there is a growing interest in the potential of mobile phones in health and chronic disease (also called mobile health or m-health). M-health includes smart phone applications (apps), texting and social media to connect to healthcare professionals (HCP) and/or a community, and use of body sensors to improve health outcomes [3]. There are over 1000 smart phone apps on google play and apple app store for diabetes selfmanagement [4]. Most of the apps are specific for type-2 diabetes prevention and management (mostly an adult disease), with a few for T1DM. Apps for T1DM have various functions like logging BG values, giving BG graph analysis (which serve as feedback to promote reflection and behavioral change), setting reminders for insulin injection/BG checks/ exercise, carbohydrate counting and insulin dose calculator, diabetes education and connecting to HCP via internet [5]. Some continuous glucose monitoring sensors and glucometers automatically connect to mobile phone apps

Rajni Sharma

drrajnisharma@yahoo.com

1 Division of Pediatric Endocrinology, Department of Pediatrics, All India Institute of Medical Sciences, New Delhi 110029 India and help in logging BG or giving updates to parents/HCP. A systematic review of smart phone apps in T1DM involving both adults and children analyzed 9 studies involving standalone apps (without texting) and one-third of them showed significant improvement in $\mathrm{HbA1c}$ while another one-third showed increased adherence to BG monitoring [6]. The addition of texting/feedback did not seem to add to any further benefit in improving $\mathrm{HbAlc}$ (though there was no study directly comparing the two) [6]. There are few studies in children; a randomized controlled trial and another retrospective study showed increased adherence to self-monitoring of BG but no significant improvement in $\mathrm{HbA1c}$ values $[7,8]$ whereas another cross-over trial found reduction in Hbalc after a 3mo period [9].

In this issue of the journal, Pramanik et al. have examined the use of an indigenous diabetes app, DMCare in a beforeand-after study in a group of Indian adolescents with poorly controlled T1DM [10]. This was a stand-alone app and did not require internet connectivity after downloading. The app acted as a BG $\log$ and gave reminders for insulin shots, food and exercise. Glycemic control in the form of $\mathrm{HbAlc}$ significantly improved 3 mo after the intervention. The authors conclude that this intervention may benefit adolescents with T1DM in achieving glycemic targets.

So should we recommend mobile apps in routine clinical practice to adolescents with T1DM? The study by Pramanik et al. was limited by lack of a control group, small sample size and short duration of follow-up [10]. Further, the continual usage or engagement of users with various apps remains an issue in the long term [4]. The use of smart phones is also limited by availability and affordability. The patient population that would benefit the most from smart phone interventions still needs to be defined. Numerable apps are available for use but their efficacy and safety needs to be evaluated in clinical studies before recommendation and regulatory authorities may have a role to play [3]. Safety of these apps has come under scrutiny especially for the ones used as insulin bolus dose calculators [11]. 
In conclusion, there is evidence to show that smartphone apps have a role in diabetes self-management and are useful adjuncts to standard clinical care in T1DM, at least in the short-term. However, long-term engagement and efficacy in improving glycemic control, preventing chronic complications and improving quality of life need to be established by future research.

\section{Compliance with Ethical Standards}

Conflict of Interest None.

\section{References}

1. Pihoker C, Forsander G, Fantahun B, et al. ISPAD Clinical Practice Consensus Guidelines 2018: The delivery of ambulatory diabetes care to children and adolescents with diabetes. Pediatr Diabetes. 2018;19:84-104.

2. DCCT Research Group (Diabetes Control and Complications Trial Research Group). The effect of intensive treatment of diabetes on the development and progression of long-term complications in insulin-dependent diabetes mellitus. N Engl J Med. 1993;329: 977-86.

3. Sim I. Mobile devices and health. N Engl J Med. 2019;381:956-68.

4. Burki TK. Mobile apps and metabolic health. Lancet Diabetes Endocrinol. 2017;5:17.
5. Sheehy S, Cohen G, Owen KR. Self-management of diabetes in children and young adults using technology and smartphone applications. Curr Diabetes Rev. 2014;10:298-301.

6. Sun C, Malcolm JC, Wong B, Shorr R, Doyle MA. Improving glycemic control in adults and children with type 1 diabetes with the use of smartphone-based mobile applications: a systematic review. Can J Diabetes. 2019;43:51-8.e3.

7. Goyal S, Nunn CA, Rotondi M, et al. A mobile app for the selfmanagement of type 1 diabetes among adolescents: a randomized controlled trial. JMIR Mhealth Uhealth. 2017;5:e82.

8. Clements MA, Staggs VS. A mobile app for synchronizing glucometer data: impact on adherence and glycemic control among youths with type 1 diabetes in routine care. J Diabetes Sci Technol. 2017;11:461-7.

9. Klee P, Bussien C, Castellsague M, et al. An intervention by a patient-designed do-it-yourself mobile device app reduces $\mathrm{HbA} 1 \mathrm{c}$ in children and adolescents with type 1 diabetes: a randomized double-crossover study. Diabetes Technol Ther. 2018;20:797-805.

10. Pramanik BK, Angelin JJ, Mathai VJ, Mathai S, Korula S, Simon A. Smartphone app as motivational intervention to improve glycemic control in adolescents with type 1 diabetes. Indian J Pediatr. 2019. https://doi.org/10.1007/s12098-019-03035-x.

11. Hirsch IB, Parkin CG. Unknown safety and efficacy of smartphone bolus calculator apps puts patients at risk for severe adverse outcomes. J Diabetes Sci Technol. 2016;10:977-80.

Publisher's Note Springer Nature remains neutral with regard to jurisdictional claims in published maps and institutional affiliations. 